\title{
Avaliação dos efeitos do Pygeum africanum rosaceae sobre a fertilidade de ratos
}

\author{
Evaluation of the effect of Pygeum africanum rosaceae in rat fertility \\ João Roberto Braga de Mello', Jussara Zani Maia², Angela Pötter de Castro² \\ \& Fernanda Bastos de Mello ${ }^{3}$
}

\begin{abstract}
RESUMO
Pygeum africanum é uma planta usada como fitoterápico no tratamento de distúrbios da micção provocados por hiperplasia prostática benigna, principalmente em seres humanos e cães. Este trabalho teve por objetivo avaliar a toxicidade reprodutiva do extrato da casca de P. africanum, administrado em ratos Wistar. Os machos foram tratados por 91 dias e as fêmeas por 77 dias, no período pré-acasalamento, gestação e lactação, com sonda orogástrica, nas dosagens de 1,5 mg/kg, 4,5 mg/kg e $15 \mathrm{mg} / \mathrm{kg}$. O grupo controle (C) recebeu salina em volume idêntico aos demais grupos (10 mL/kg). Não foram observadas alterações nos animais tratados com qualquer das três dosagens de $P$. africanum em comparação com os do grupo controle, quanto ao desenvolvimento ponderal, consumo de água e ração. Não foram detectadas alterações morfológicas nos órgãos internos, cuja massa relativa não diferiu do controle. A avaliação do líquido seminal do ducto deferente e dos testículos não mostrou diferenças na morfologia espermática e na contagem de células espermáticas, comparando o grupo controle com qualquer das dosagens de P. africanum. Não houve interferência sobre a gestação, com as ratas parindo filhotes em número e massa corporal normal. Os índices reprodutivos e de desenvolvimento pós-natal não apresentaram alterações significativas com qualquer das dosagens de P. africanum em relação ao grupo controle. Os filhotes de animais tratados com o P. africanum apresentaram desenvolvimento pós-natal semelhante aos filhotes do grupo controle, com aparecimento de características de desenvolvimento sexual dentro do período previsto para a espécie. Os resultados permitem concluir que a administração do P. africanum, em dosagens até 10 vezes a usada com finalidade terapêutica, não interfere negativamente na reprodução de ratos. O fitoterápico pode ser considerado atóxico sobre a reprodução de ratos.
\end{abstract}

Descritores: Pygeum africanum, toxicidade reprodutiva, fitoterápico.

\begin{abstract}
Pygeum africanum as a phytotherapic agent is used to treat human and canine mictoric disturbances observed in benign prostate hypertrophy. In these study we investigated the potential reproductive toxicity of a Pygeum africanum bark extract in Wistar rats. The so called Segment I of FDA was performed and the mature male rats were orally treated over a period of 91 days, while the female rats were treated during the pre-mating, mating, pregnancy and lactation periods (77 days). The animals received daily per os $1.5 \mathrm{mg} / \mathrm{kg}, 4.5 \mathrm{mg} / \mathrm{kg}$ and $15 \mathrm{mg} / \mathrm{kg}$ of the extract. A control group received saline in the same volume $(10 \mathrm{~mL} / \mathrm{kg})$. The daily evaluation of body weight, water and food consumption did not show any difference between the Pygeum africanum groups and the control. No morphological alteration on visceral organ was observed, and the relative weight was similar to the control. The seminal fluid evaluation didn't show differences between Pygeum africanum groups and control in spermatic count and morphology. The pregnancy was not affected and the dams gave birth to normal litters. The reproductive and post-natal index didn't show significant differences between groups. The general and sexual development of the Pygeum africanum offspring was normal, considering the specie. It is concluded that the administration of Pygeum africanum up to 10 times the proposed therapeutic dose did not affect rat reproduction. The phytotherapic can be considered non toxic in rat reproduction.
\end{abstract}

Key words: Pygeum africanum, reproductive toxicity, phytotherapic.

${ }^{1}$ Departamento de Farmacologia, Instituto de Ciências Básicas da Saúde (ICBS), Universidade Federal do Rio Grande do Sul (UFRGS). ${ }^{2}$ Programa de Pós-graduação em Ciências Veterinárias (PPGCV) - UFRGS. CORRESPONDÊNCIA: J.R.B.Mello [jmello@vortex.ufrgs.br; FAX: +55 51 3316 $3121]$. 


\section{INTRODUÇÃO}

O uso de plantas medicinais como recurso terapêutico em medicina humana vem sendo assimilado na terapêutica veterinária. Nos países em desenvolvimento o uso chega a atingir $85 \%$ do total de substâncias usadas em terapêutica [22]. Uma das razões para essa popularidade é a falsa percepção de que estes medicamentos são seguros e livres de efeitos adversos [8]. A existência de poucos estudos quanto à ação biológica e efeitos tóxicos, tornam a utilização de fitoterápicos um assunto polêmico, onde prepondera a inexistência de comprovação de eficácia e segurança toxicológica $[10,22,23]$.

A eficácia e aceitabilidade da utilização do extrato de $P$. africanum (ameixa africana) no tratamento de desordens de micção associadas à hiperplasia prostática benigna (HPB), bem como na redução da hiperplasia e adenoma prostático, foi testada e comprovada em experimentos multicêntricos $[1,3,9,13]$. No Brasil a utilização do extrato de $P$. africanum para tal fim também é crescente $[4,15]$. Visto se tratar de um fitoterápico de uso contínuo, sem relatos sobre avaliação toxicológica, especialmente toxicidade crônica e reprodutiva.

O presente trabalho avaliou os efeitos da administração crônica de três dosagens do extrato de $P$. africanum sobre a fertilidade de ratos, as possíveis alterações no desenvolvimento das progênies expostas durante a gestação e lactação; avaliar o desenvolvimento das progênies e toxicidade sistêmica dos animais submetidos à administração crônica do fitoterápico. Foi utilizado o "segmento I", recomendado pela Food and Drug Administration (FDA) e Organization for Economic Cooperation and Development (OECD) para investigação de substâncias sobre a reprodução.

\section{MATERIAIS E MÉTODOS}

O extrato da casca de Pygeum africanum usado nos experimentos foi fornecido pelo Laboratório Galenogal Farma, responsável pela garantia de pureza do produto testado. Foi utilizada formulação granulada na concentração de $100 \mathrm{mg}$ de $P$. africanum por grama de granulado, contendo como excipientes lactose, amido de milho, polividona, estearato de magnésio e talco. $\mathrm{O}$ produto pertencia ao lote piloto n. 99001 de procedência da Importadora Indena, lote 18.003. Como animais experimentais foram utilizados 36 ratos e 108 ratas albinos da raça Wistar, com idade aproximada de 120 dias, criados e mantidos no Biotério do Departamento de Farmacologia do Instituto de Ciências Básicas da Saúde da UFRGS, com condições constantes de umidade, temperatura $\left(21^{\circ} \mathrm{C} \pm 2\right)$ e ciclo de claro/escuro de 12 horas (claro das 9 às $21 \mathrm{~h}$ ), alimentados com ração comercial $^{1}$ e água ad libitum durante todo o período experimental.

Os animais foram divididos aleatoriamente em quatro grupos experimentais, contendo entre oito e dez machos e vinte e quatro a trinta fêmeas cada grupo. Todos receberam tratamento diário per os com auxílio de sonda oro-gástrica, nas dosagens: (a) grupo $P$. africanum dosagem diária de $1,5 \mathrm{mg} / \mathrm{kg}$ de massa corporal (PA 1,5), equivalente à dose total diária recomendada para seres humanos [16]; (b) grupo $P$. africanum dosagem diária de $4,5 \mathrm{mg} / \mathrm{kg}$ de massa corporal (PA 4,5), equivalente à três vezes a dosagem GPA1; (c) grupo P. africanum dosagem diária de $15 \mathrm{mg} / \mathrm{kg}$ de massa corporal (PA 15), equivalente à dez vezes a dosagem GPA1 e (d) grupo controle (C), tratado com solução fisiológica na dosagem de $10 \mathrm{~mL} /$ $\mathrm{kg}$ de massa corporal, volume equivalente aos demais grupos.

Os machos foram tratados por um período de 91 dias (70 dias antes do acasalamento e 21 dias de acasalamento). As fêmeas foram tratadas por 14 dias prévios ao acasalamento, durante o acasalamento, gestação e lactação, perfazendo um período máximo de 77 dias. O período de acasalamento teve duração de 21 dias, correspondendo a três ciclos de cinco dias consecutivos, com intervalo de 48 horas entre cada um dos ciclos. Na caixa individual de cada macho foram introduzidas três fêmeas virgens, permanecendo com eles durante as duas últimas horas do período escuro (entre 7 e 9 h). Após esse período, os animais foram separados e a constatação da prenhez foi realizada mediante a avaliação da presença de espermatozóides nos esfregaço vaginal, realizado diariamente e analisado ao microscópio óptico com aumento de 10 vezes. Vinte quatro horas após a constatação do esfregaço vaginal positivo foi considerado o primeiro dia de gestação. As variáveis avaliadas nos ma- 
chos foram (a) massa corporal diária (g) durante 91 dias; (b) consumo diário de ração (g) e água (ml); (c) peso relativo (\%) das vísceras ao final do acasalamento (fígado, baço, rins, coração, vesícula seminal, testículo, próstata e epidídimo); (d) contagem do número total de espermatozóides, produção diária de espermatozóides e percentual de alterações morfológicas nos mesmos; (e) aparecimento de sinais de toxicidade sistêmica. Nas fêmeas foram avaliados (a) massa corporal diária (g) durante o préacasalamento, acasalamento, gestação e lactação; (b) consumo diário de ração $(\mathrm{g})$ e água $(\mathrm{ml})$; (c) peso relativo (\%) das vísceras ao final da lactação (fígado, rins, baço, coração, ovários e útero); (d) sinais de aborto, distocias e prolongamento da gestação; (e) número de implantes uterinos e (f) taxas reprodutivas (acasalamento, gestação, natalidade, viabilidade, desmame, alterações macroscópicas fetais, perdas pós-implantação). Nos filhotes foram avaliados (a) alterações macroscópicas externas ao nascer; (b) número de filhote por ninhada; (c) percentual de machos e fêmeas; (d) número de filhotes nascidos vivos e mortos; (e) peso individual ao nascer, aos 7, 14 e 21 dias e (f) desenvolvimento de características gerais e sexuais dos filhotes. Os dados foram analisados por análise de variância ou, alternativamente, pelo teste de Kruskal-Wallis, sempre que a distribuição dos dados não era normal. $\mathrm{O}$ teste de Tukey foi usado para identificar diferenças entre grupos. As porcentagens foram analisadas pelo teste Qui-quadrado ou, alternativamente, pelo teste exato de Fischer. A avaliação estatística foi realizada com a utilização dos programas Minitab e Excel. Adotou-se como estatisticamente significativo o nível de $5 \%$ [11].

\section{RESULTADOS}

Não foram observadas alterações significativas nos ratos tratados com qualquer das três dosagens de Pygeum africanum em comparação com os animais do grupo controle quanto ao desenvolvimento ponderal ou no consumo de água e ração durante todo o experimento. As flutuações em torno do normal não fugiram às médias registradas pelo Departamento de Farmacologia da UFRGS, nem para os animais tratados com $P$. africanum nem com os do grupo controle. Não foram detectadas alterações morfológicas nos órgãos internos, cuja massa relativa não diferiu significativamente do controle, com qualquer das dosagens de $P$. africanum avaliadas (Tabela 1).

Tabela 1. Massa relativa dos órgãos (\%) de ratos tratados durante 91 dias consecutivos com extrato de Pygeum africanum 1,5; 4,5 e $15 \mathrm{mg} / \mathrm{kg}$ de massa corporal, e grupo controle (C), tratado com solução fisiológica na dosagem de $10 \mathrm{ml} / \mathrm{kg}$. Os valores são apresentados como médias \pm erro padrão da média.

\begin{tabular}{ccccc}
\hline Massa relativa dos & \multirow{2}{c}{ órgãos (\%) } & & \multicolumn{3}{c}{ Pygeum africanum } \\
\cline { 3 - 5 } & $\mathrm{n}=10$ & $\begin{array}{c}1,5 \mathrm{mg} / \mathrm{kg} \\
\mathrm{n}=8\end{array}$ & $\begin{array}{c}4,5 \mathrm{mg} / \mathrm{kg} \\
\mathrm{n}=8\end{array}$ & $\begin{array}{c}15 \mathrm{mg} / \mathrm{kg} \\
\mathrm{n}=10\end{array}$ \\
\hline Coração & $0,38 \pm 0,01$ & $0,35 \pm 0,01$ & $0,36 \pm 0,02$ & $0,34 \pm 0,03$ \\
Baço & $0,15 \pm 0,01$ & $0,16 \pm 0,01$ & $0,14 \pm 0,01$ & $0,14 \pm 0,005$ \\
Fígado & $3,48 \pm 0,08$ & $3,50 \pm 0,08$ & $3,51 \pm 0,1$ & $3,53 \pm 0,1$ \\
Rim direito & $0,32 \pm 0,01$ & $0,30 \pm 0,01$ & $0,31 \pm 0,01$ & $0,31 \pm 0,007$ \\
Rim esquerdo & $0,32 \pm 0,006$ & $0,30 \pm 0,01$ & $0,31 \pm 0,01$ & $0,31 \pm 0,007$ \\
Testículo direito & $0,36 \pm 0,01$ & $0,37 \pm 0,01$ & $0,35 \pm 0,01$ & $0,34 \pm 0,01$ \\
Testículo esquerdo & $0,37 \pm 0,01$ & $0,37 \pm 0,01$ & $0,35 \pm 0,01$ & $0,34 \pm 0,01$ \\
Epidídimo direito & $0,15 \pm 0,006$ & $0,15 \pm 0,005$ & $0,15 \pm 0,006$ & $0,15 \pm 0,005$ \\
Epidídimo esquerdo & $0,15 \pm 0,003$ & $0,15 \pm 0,004$ & $0,15 \pm 0,005$ & $0,15 \pm 0,004$ \\
Vesícula seminal & $0,14 \pm 0,004$ & $0,14 \pm 0,006$ & $0,14 \pm 0,006$ & $0,14 \pm 0,008$ \\
Próstata & $0,13 \pm 0,01$ & $0,12 \pm 0,01$ & $0,13 \pm 0,01$ & $0,11 \pm 0,01$ \\
\hline $\mathrm{n}$ número de animais por grupo & & & \\
\end{tabular}


O exame do líquido seminal do ducto deferente e dos testículos não mostrou diferenças na morfologia espermática ou na contagem de células espermáticas, comparado ao controle com qualquer das dosagens de P. africanum (Tabela 2).

As ratas tratadas durante o pré-acasalamento, acasalamento, gestação e amamentação toleraram bem a administração de $P$. africanum, não sendo observadas alterações comportamentais, de ambulação e respiração que indicassem toxicidade do fitoterápico.

Não foram detectadas alterações morfológicas nos órgãos internos, cuja massa relativa não diferiu significativamente do controle, com qualquer das dosagens de $P$. africanum avaliadas (Tabela 3).
Não houve interferência significativa sobre a gestação. As ratas pariram no prazo previsto, típico da espécie ( 21 a 22 dias), filhotes em número e massa corporal normal, não significativamente diferente das fêmeas usadas como controle. Não se verificaram significativas alterações no desenvolvimento ponderal, no consumo de água e ração em qualquer das dosagens de $P$. africanum quando comparados com o grupo controle.

Os índices reprodutivos e de desenvolvimento pós-natal não apresentaram alterações significativas com qualquer das dosagens de $P$. africanum em relação ao grupo controle (TAB. 4).

O desenvolvimento físico pós-natal das proles nascidas de ratas tratadas durante o pré-acasalamento,

Tabela 2. Índices de fertilidade dos ratos tratados durante 91 dias consecutivos com extrato de Pygeum africanum 1,5; 4,5 e $15 \mathrm{mg} / \mathrm{kg}$ de massa corporal, e grupo controle (C), tratado com solução fisiológica na dosagem de $10 \mathrm{ml} / \mathrm{kg}$. Os valores são apresentados como médias \pm erro padrão da média.

\begin{tabular}{|c|c|c|c|c|}
\hline & \multirow{2}{*}{$\mathrm{C}=10$} & \multicolumn{3}{|c|}{ Pygeum africanum } \\
\hline & & $\begin{array}{c}1,5 \mathrm{mg} / \mathrm{kg} \\
\mathrm{n}=8\end{array}$ & $\begin{array}{c}4,5 \mathrm{mg} / \mathrm{kg} \\
\mathrm{n}=8\end{array}$ & $\begin{array}{c}15 \mathrm{mg} / \mathrm{kg} \\
\mathrm{n}=10\end{array}$ \\
\hline Produção diária de espermatozóides (x 106)\# & $27,2 \pm 2,1$ & $22,9 \pm 3,8$ & $24,3 \pm 3,1$ & $28,1 \pm 4,1$ \\
\hline Número de espermatozóides $(\times 106)^{*}$ & $176,7 \pm 27,1$ & $180,6 \pm 30,2$ & $172,2 \pm 20,2$ & $204,5 \pm 34,1$ \\
\hline Espermatozóides anormais (\%) & $5,7 \pm 0,8$ & $6,2 \pm 1,2$ & $6,3 \pm 1,4$ & $5,6 \pm 1,0$ \\
\hline \multicolumn{5}{|c|}{$\begin{array}{l}\text { \# A produção diária de espermatozóides foi medida a partir da contagem de células espermáticas em câmara de Neubauer, a parti } \\
\text { de macerados de testículos [17]. } \\
\text { "O número de espermatozóides foi determinado a partir de sua contagem em alíquota do macerado da cauda do epidídimo en } \\
\text { câmara de Neubauer [17]. }\end{array}$} \\
\hline
\end{tabular}

Tabela 3. Massa relativa dos órgãos $(\%)$ de ratas tratadas diariamente durante o pré-acasalamento, acasalamento, gestação e lactação com extrato de Pygeum africanum 1,5; 4,5 e $15 \mathrm{mg} / \mathrm{kg}$ de massa corporal, e grupo controle (C), tratado com solução fisiológica na dosagem de $10 \mathrm{~mL} / \mathrm{kg}$. Os valores são apresentados como médias \pm erro padrão da média.

\begin{tabular}{ccccc}
\hline Massa relativa & GC & \multicolumn{3}{c}{ Pygeum africanum } \\
\cline { 3 - 5 } dos órgãos (\%) & & $\begin{array}{c}1,5 \mathrm{mg} / \mathrm{kg} \\
\mathrm{n}=24\end{array}$ & $\begin{array}{c}4,5 \mathrm{mg} / \mathrm{kg} \\
\mathrm{n}=24\end{array}$ & $\begin{array}{c}15 \mathrm{mg} / \mathrm{kg} \\
\mathrm{n}=30\end{array}$ \\
\hline Coração & $0,40 \pm 0,007$ & $0,39 \pm 0,01$ & $0,38 \pm 0,02$ & $0,41 \pm 0,02$ \\
Baço & $0,17 \pm 0,01$ & $0,16 \pm 0,01$ & $0,17 \pm 0,01$ & $0,18 \pm 0,01$ \\
Fígado & $5,21 \pm 0,22$ & $4,50 \pm 0,18$ & $4,51 \pm 0,10$ & $4,54 \pm 0,17$ \\
Rim direito & $0,38 \pm 0,006$ & $0,36 \pm 0,01$ & $0,36 \pm 0,01$ & $0,37 \pm 0,003$ \\
Rim esquerdo & $0,38 \pm 0,01$ & $0,37 \pm 0,01$ & $0,37 \pm 0,01$ & $0,36 \pm 0,008$ \\
Ovário direito & $0,01 \pm 0,001$ & $0,01 \pm 0,001$ & $0,01 \pm 0,001$ & $0,01 \pm 0,002$ \\
Ovário esquerdo & $0,01 \pm 0,001$ & $0,01 \pm 0,001$ & $0,01 \pm 0,001$ & $0,01 \pm 0,001$ \\
Útero & $0,08 \pm 0,04$ & $0,09 \pm 0,01$ & $0,09 \pm 0,01$ & $0,11 \pm 0,01$ \\
\hline
\end{tabular}


Tabela 4. Índices de fertilidade e taxas reprodutivas de ratas tratadas diariamente durante o pré-acasalamento, acasalamento, gestação e lactação com extrato de Pygeum africanum 1,5; 4,5 e $15 \mathrm{mg} / \mathrm{kg}$ de massa corporal, e grupo controle (C), tratado com solução fisiológica na dosagem de $10 \mathrm{~mL} / \mathrm{kg}$. São apresentados valores médios \pm erro padrão da média. As taxas são apresentadas em porcentagem.

\begin{tabular}{ccccc}
\hline Índices de fertilidade e taxas reprodutivas & $\mathrm{C}$ & \multicolumn{3}{c}{ Pygeum africanum } \\
\cline { 3 - 5 } & & $1,5 \mathrm{mg} / \mathrm{kg}$ & $4,5 \mathrm{mg} / \mathrm{kg}$ & $15 \mathrm{mg} / \mathrm{kg}$ \\
\hline Fêmeas prenhes & 7 & 8 & 11 & 8 \\
Filhotes & 72 & 70 & 124 & 77 \\
Filhotes/ninhada & $10,3 \pm 0,01$ & $8,85 \pm 2,01$ & $11,3 \pm 1,07$ & $9,62 \pm 1,27$ \\
Peso do filhote ao nascer (g) & $6,71 \pm 0,02$ & $6,17 \pm 0,09$ & $5,47 \pm 0,38$ & $6,20 \pm 0,07$ \\
Taxa de natalidade & 97,8 & 90,0 & 95,2 & 92,0 \\
Taxa de viabilidade & 95,6 & 98,4 & 96,6 & 91,0 \\
Taxa de desmame & 98,0 & 98,4 & 95,8 & 98,0 \\
Taxa de gestação & 90 & 89,5 & 95,8 & 100 \\
Taxa de perdas pós-implantação & 8 & 9 & 8 & 7 \\
Alterações macroscópicas fetais & Nihil & Nihil & Nihil & Nihil \\
\hline
\end{tabular}

Taxa de natalidade $=\mathrm{n}^{\mathrm{o}}$ de filhotes nascidos vivos / no de filhotes nascidos X 100.

Taxa de viabilidade $=\mathrm{n}^{\mathrm{o}}$ de filhotes vivos até o $4^{\mathrm{o}}$ dia de lactação $/ \mathrm{n}^{\mathrm{o}}$ de filhotes nascidos vivos X 100.

Taxa de desmame $=$ no de filhotes ao desmame $/ \mathrm{n}^{\mathrm{o}}$ de filhotes nascidos vivos $\mathrm{X} 100$.

Taxa de gestação $=n^{0}$ de fêmeas prenhes / no de fêmeas com espermatozóides no esfregaço vaginal X 100 .

Taxa de perdas pós-implantação $=$ no $^{\circ}$ de sítios de implantação - no de fetos nascidos / no de sítios de implantação X 100 .

acasalamento, gestação e lactação não mostrou diferença em relação às proles das ratas controles, até a idade de 21 dias, ocasião do desmame.

A idade no surgimento das características de desenvolvimento sexual das progênies expostas a dosagens crescentes de Pygeum africanum durante as fases pré e perinatal, descida dos testículos, separação prepucial e abertura do canal vaginal não revelaram diferenças significativas entre os grupos e em comparação com o controle. A descida dos testículos ocorreu entre o décimo terceiro e décimo sexto dia pós-natal em todos os grupos. A separação prepucial ocorreu entre o vigésimo quinto e trigésimo quinto dia após o parto. A abertura do canal vaginal ocorreu entre o trigésimo primeiro e quadragésimo primeiro dias pós-natal. Esses dados não diferem daquelas registradas pelo Departamento de Farmacologia da UFRGS para o aparecimento dessas características.

\section{DISCUSSÃO}

Por definição, índice terapêutico (IT) representa a relação entre a dose letal para 50\% dos indivíduos (DL50) e a dose eficaz para 50\% dos indivíduos (DE50). Para uma maior segurança farmacológica, uma definição mais conservadora é a que relaciona a dose letal para $1 \%$ dos indivíduos com a dose eficaz para $99 \%$ dos indivíduos, ou ainda a dose capaz de provocar efeitos adversos (dose LOEL) em relação à maior dose incapaz de produzir efeitos adversos (dose NOEL). A relação de 10 vezes, em qualquer dos casos, representa uma relativa segurança para a substância química ou para o fitoterápico, ou baixa toxicidade [20,21]. Considerando que as dosagens diárias usadas do extrato de Pygeum africanum nesse experimento de $1,5 \mathrm{mg} / \mathrm{kg}, 4,5 \mathrm{mg} / \mathrm{kg}$ e $15 \mathrm{mg} /$ $\mathrm{kg}$ de massa corporal representam, respectivamente uma, três e dez vezes a dosagem recomendada para o tratamento de distúrbios de micção associados a hiperplasia prostática benigna e na redução da hiperplasia e adenoma prostático [16], e considerando ainda que os animais foram tratados por um período de aproximadamente 90 dias, o fitoterápico pode ser considerado atóxico [21].

Os sinais de toxicidade sistêmica são definidos a partir da redução na massa corporal dos animais experimentais [2]. Além da redução do desenvolvimento ponderal, a toxicidade sistêmica se manifesta através da redução nos consumos de água e ração, alterações comportamentais como prostração, apatia e má condição da pelagem, com presença de pelos arrepiados $[17,18]$. Outros sinais de toxicidade podem se expressar pela alteração da massa relativa dos ór- 
gãos [5]. Os resultados obtidos não mostram qualquer alteração nas variáveis relacionadas com a toxicidade sistêmica com a utilização de $P$. africanum em dosagens até 10 vezes maiores do que as preconizadas para seres humanos. Testes de toxicidade aguda usando dosagens de 1 a $8 \mathrm{~g} / \mathrm{kg}$ em ratos e 1 a $6 \mathrm{~g} / \mathrm{kg}$ em camundongos, não determinaram mortes dentro de 48 horas [19]. O mesmo autor avaliou a toxicidade crônica do $P$. africanum em dosagens de 60 a $600 \mathrm{mg} / \mathrm{kg}$ durante 11 meses, não observando qualquer efeito adverso. A presença de dor abdominal e aumento do peristaltismo intestinal como efeitos adversos do Pygeum africanum foram observados em seres humanos utilizando dosagens mais baixas que as usadas neste trabalho [1,9]. Isso pode indicar uma susceptibilidade da espécie, o que pode limitar a transposição de dados uma espécie para outra [21].

As substâncias tóxicas administradas por via oral podem ultrapassar a barreira testicular e serem biotransformadas nas gônadas. A substância tóxica ou o produto de sua biotransformação podem resultar em compostos que atuam diretamente sobre os tipos celulares existentes no testículo, interferindo na espermatogênese e/ou esteroidogênese [24]. O mesmo autor sugere que alterações como redução no número e na produção diária de espermatozóides podem ter origem em lesões provocadas no epitélio germinativo. Anormalidades espermáticas também prejudicam seu transporte no útero, o que aumenta a probabilidade de redução da fertilidade [6]. Os resultados obtidos com relação à fertilidade dos machos revelam a inexistência de toxicidade reprodutiva para os ratos tratados com P. africanum.

A avaliação das taxas reprodutivas de natalidade, viabilidade, desmame, gestação, perdas pós-implantação, entre outras, proporcionam em conjunto um ótimo indicativo de aferição de interferência de xenobióticos sobre a reprodução [21]. A interpretação detalhada de alterações nas taxas reprodutivas pode identificar o período no qual os efeitos tóxicos reprodutivos se estabeleceram [12]. No presente trabalho não foram observadas diferenças estatisticamente significativas nas taxas reprodutivas de animais submetidos à administração das três dosagens de $P$. africanum, confirmando a ausência de toxicidade reprodutiva para a espécie.
Com relação ao desenvolvimento ponderal das proles de fêmeas tratadas, a análise estatística não revelou diferença significativa. $\mathrm{O}$ peso das ninhadas pode ser influenciado pelo nível de crescimento intrauterino, pelo tamanho da ninhada e pelo tempo de gestação. O peso da prole durante o aleitamento é dependente de fatores como peso ao nascer, habilidade da fêmea e efeitos tóxicos de substâncias. Alterações neste sentido fazem com que a viabilidade fetal seja alterada com relação aos controles [6].

A utilização de plantas que interferem no desenvolvimento ponderal dos filhotes pode retardar o aparecimento de características de desenvolvimento geral e sexual dos animais [7]. No caso do P. africanum, os eventos de avaliação de desenvolvimento geral não sofreram modificações quando comparados com os animais do grupo controle. A descida de testículos à bolsa escrotal, a separação prepucial e a abertura do canal vaginal são eventos hormônio-dependente e qualquer alteração responsável por interferências na produção hormonal pode repercutir na manifestação dessas características [14]. Não há, entre os resultados, indicativo de interferência do extrato de $P$. africanum no desenvolvimento sexual das proles.

\section{CONCLUSÕES}

Não há, entre os resultados obtidos, indicações de que o extrato da casca de Pygeum africanum possa causar efeitos tóxicos sobre a reprodução de ratos Wistar quando administrado em dosagem até 10 vezes acima da indicada para uso terapêutico em seres humanos.

Considerando que o índice terapêutico é a relação entre DL50 e DE50, pode-se concluir que o índice do extrato da casca de $P$. africanum para ratos é maior que 10 .

Os resultados permitem concluir que o extrato da casca de $P$. africanum se enquadra na categoria de fitoterápico com praticamente atóxico e/ou relativamente não prejudicial.

\section{Nota Informativa}

${ }^{1}$ Nuvilab CR1 - Nuvital, Colombo/PR. 


\section{REFERÊNCIAS}

1 Barlet A., Albrecht J., Aubert A., Fischer M., Grof F., Grothuesmann H.G., Masson J.C., Mazeman E. Mermon R., Reichelt H., Schönmetzler F. \& Suhler A. 1990. Wirksamkeit eines extraktes aus Pygeum africanum in der medikamentösen therapie von miktionsstörumgen infolge einer benignen prostathyperplasie: Bewerbung objektiver und subbektiver parameters - Eine placebokontrollierte doppelblide multizenterstudie. Wiener Klinische Wochensschrift.102: 667-672.

2 Black D.L. \& Marks T.A. 1992. Role of maternal toxicity in assessing developmental toxicity in animals: a discussion. Regulatory Toxicology and Pharmacology. 16: 189-202.

3 Breza J., Dzurny O., Borowka A., Hanus T., Petrik R., Blane G. \& Chad-Boreham H. 1998. Efficacy and acceptability of Tadenanâ (Pygeum africanum) in the treatment of benign prostatic hyperplasi (BPH): A multicenter trial in central Europe. Current Medic Research and Opinion. 14: 127-139.

4 Castro A.P. 2002. Avaliação toxicológica dos fitoterápicos Ginkgo biloba e Pygeum africanum sobre a fertilidade e reprodução de ratos Wistar. 119 f. Porto Alegre, RS. Dissertação (Mestrado em Ciências Veterinárias) - Programa de Pósgraduação em Ciências Veterinárias, Universidade Federal do Rio Grande do Sul.

5 Dallegrave E. 2003. Toxicidade reprodutiva do herbicida glifosato - Roundupâ em ratos Wistar. 225 f. Tese (Doutorado em Ciências Veterinárias) - Programa de Pós-graduação em Ciências Veterinárias, Universidade Federal do Rio Grande do Sul.

6 EPA - Enviromental Protection Agency. Reprodution and fertility effects. 1996. In: Health effects test guidelines. 570p. (OPPTS 870: 3800).

7 Gerenutti M., Spinosa H.S. \& Bernardi M.M. 1992. Effects of bracken fern (Pteridium aquilinum L. Kuhn) feeding during the development of female rats and their offspring. Veterinary Human Toxicology. 34: 307-310.

8 Hamid S., Rojter S. \& Vierling J. 1997. Prostacted cholestasis hepatitis after use of Prostataâ. Annals of Urology. 127: 169-170.

9 Krzeski T., Kazón M., Borkowski A., Witeska A. \& Kuczera J. 1993. Combined extracts of Urtica dioica and Pygeum africanum in the treatment of benign prostatic hiperplasia Double-blind comparison of two doses. Clinical Therapeutics. 15: 1011-1020.

10 Lapa A.J. 1999. Farmacologia e toxicologia de produtos naturais. In: Simões C.M.O. (Ed). Farmacognosia da planta ao medicamento. Florianópolis: Editora da Universidade Federal de Santa Catarina, pp.181-196.

11 Lapponi J.C. 2000. Estatística usando o Excel. São Paulo: Lipponi , 451p.

12 Lemonica I.P., Damasceno D.C. \& Di-Stasi L.C. 1996. Study of embryotoxic effects of an extract of rosemary (Rosmarinus officinalis L.) Brazilian Journal of Medical and Biological Research. 29: 223-227.

13 Levin R.M., Riffaud J.P., Bellamy F., Rohrmann D., Kranspolsky L., Haugaard N., Zhao Y. \& Wein A.J. 1996. Effects of Tadenan pretreatment on bladder physiology and biochemistry following partial outlet obstrution. The Journal of Urology. 156: 2084-2088.

14 Mably T.A., Moore R.W., Goy R.W. \& Peterson R.E. 1992. In utero and lactational exposure of male rats to 2,3,7,8 tetrachlorobenzene-p-dioxin. 3. Effects on sexual behaviour and regulation of lutheinizing hormone secretion in adulthood. Toxicology and Applied Pharmacology. 114: 108-117.

15 Maia J.Z. 2001. Estudo do efeito da administração crônica de Pygeum africanum sobre o desenvolvimento ponderal e a fertilidade de ratos Wistar e sobre o desenvolvimento de suas progênies. $104 \mathrm{f}$. Porto Alegre, RS. Dissertação (Mestrado em Ciências Veterinárias) - Curso de Pós-graduação em Ciências Veterinárias, Universidade Federal do Rio Grande do Sul.

16 Mathé G., Orbach-Arbouys S., Bizi E. \& Court B. 1995. The so-called phytoestrogenic action of Pygeum africanum extract. Biomedicine and Pharmacotherapy. 49: 339-340.

17 Mello F.B. 2001. Estudo dos efeitos de Lantana camara (Verbenaceae) sobre a fertilidade e reprodução de ratos. 120f. Porto Alegre, RS. Dissertação (Mestrado em Ciências Veterinárias) - Programa de Pós-graduação em Ciências Veterinárias, Universidade Federal do Rio Grande do Sul.

18 Mello J.R.B., Langeloh A., Habermehl G., Krebs H.C., Batatinha M.J.M., Almeida C.C.R., Bastos F.C., Bassani M., Baruffaldi C., Alavares F., Francisco D. \& Kummer R. 1997. Avaliação do extrato aquoso dos frutos de Crotalaria retusa Leguminosae sobre a fertilidade de ratas. Arquivos da Faculdade de Veterinária UFRGS. 25: 34-42. 
19 Murray M.T. 2001. Pygeum africanum - A botanical review. Natural Medicine on line. http://www.nat.med.com/archives/ afrivanum.htm. Capturado em 7 de dezembro, 2001.

20 Oga S. 1996. Fundamentos de toxicologia. São Paulo: Atheneu, 515 p.

21 Osweiler G.D. 1998. Toxicologia veterinária. Porto Alegre: Artes Médicas, 526 p.

22 Sharapin N. 1996. Normatização da indústria farmacêutica. In: Anais do Seminário sobre industrialización y legislación de productos fitofarmacêuticos en iberoamérica (Guatemala, Guatemala). pp. 42-44.

23 Sonaglio D. 1987. Padronização do extrato hidroalcoólico das sumidades floridas de Achyroclines satureoides (Lam.) D.C.Compositae (Marcela). 163f. Porto Alegre, RS. Dissertação (Mestrado em Ciência Farmacêutica) - Curso de Pósgraduação em Ciência Farmacêutica, Universidade Federal do Rio Grande do Sul.

24 Thomas J.A. 1989. Toxic responses of the reproductive system. In: Klaassen C.D. (Ed). Toxicology-The Basis Science of Poison. New York: Thieme Medical, pp.547-581. 